\title{
Intraabdominal Cystic Lymphangiomas in Children: A Single Center Experience
}

\author{
Michaela Prehal ${ }^{\mathrm{a}}$, Josef Hager ${ }^{\mathrm{b}}$, Beatrice Haeussler ${ }^{\mathrm{b}}$, Thomas Mueller ${ }^{\mathrm{c}}$, Kathrin Maurer ${ }^{\mathrm{d}}$, \\ Markus Rauchenzauner ${ }^{\mathrm{a}}$, Martin Fruehwirth ${ }^{\mathrm{a}, \mathrm{e}}$
}

\begin{abstract}
Congenital intraabdominal cystic lymphangiomas (ICLs) are rare disorders. Only a minority of cases present with acute illness, the majority of patients show unspecific abdominal symptoms, and due to the slow progression of ICL the diagnosis is usually made with delay. We report our single center experience concerning ICL covering a 3-year period from 2009 to 2011. During this time three patients with a mean age of 2.8 years were first seen because of unspecific abdominal symptoms, and were identified to have an ICL, originating from the ligamentum gastrocolicum (diameter 17 $\mathrm{cm}$ ), gastrolienale (diameter $20 \mathrm{~cm}$ ) and omentum majus (diameter $7 \mathrm{~cm}$ ). The following report should emphasize the diagnostic difficulty associated with ICL presenting three patients in excellent condition, showing normal laboratory data despite a considerable persistent intraabdominal fluid accumulation. The persistence of "ascites" in parallel with the follow-up sonographies demonstrating cystic masses and internal septations led to the differential diagnosis of an ICL. To us, it seems to be important to bear in mind ICL as a relevant differential diagnosis in children with unspecific abdominal pain or non-specific ascites. This is to point out that especially the existence of septated intraabdominal fluid and cystic structures are strong signs leading to the diagnosis of an ICL. Due to this, the general use of ultrasonography (US) in children with unspecific abdominal symptoms should be recommended.
\end{abstract}

Keywords: Intraabdominal cystic lymphangioma; Ascites; Ultrasonography; Cystic masses; Internal septations

\section{Introduction}

Intraabdominal cystic lymphangiomas (ICLs) are congenital

Manuscript accepted for publication September 10, 2014

${ }^{a}$ Department of Pediatrics, St. Vinzenz Hospital, Zams, Austria

${ }^{b}$ Department of Pediatric Surgery, Medical University Innsbruck, Austria

${ }^{\mathrm{c}}$ Department of Pediatrics, Medical University Innsbruck, Austria

${ }^{\mathrm{d} D e p a r t m e n t ~ o f ~ R a d i o l o g y, ~ M e d i c a l ~ U n i v e r s i t y ~ I n n s b r u c k, ~ A u s t r i a ~}$

${ }^{\mathrm{e}}$ Corresponding Author: Martin Fruehwirth, Department of Pediatrics,

St. Vinzenz Hospital, Sanatoriumstrasse 43, Zams 6511, Austria.

Email: Martin.Fruehwirth@krankenhaus-zams.at

doi: http://dx.doi.org/10.14740/ijcp163w malformations of extended lymphatic vessels whose connection to the venous and lymphatic system are supposed to be defective $[1,2]$. An intraabdominal location of cystic lymphangiomas can be shown in $5 \%$ of all lymphangiomas corresponding to an incidence between 1:27,000 and 1:250,000 [1]. Generally, this type of lymphangioma is detected in the first decade of life, and in $60 \%$ of the cases it is diagnosed even before the fifth year of life with a significant male predominance [1, 3-5]. Clinical signs are often unspecific depending on size and location of the process ranging from acute abdominal symptoms, via chronic abdominal pain to an accidentally detected space-occupying lesion or intraabdominal fluid accumulation in asymptomatic patients $[2,3$, 6]. Even though ICL can cause acute abdominal symptoms through bowel obstruction, inflammation or intracystic hemorrhage, most of the patients present with unspecific abdominal signs like nausea, vomiting, diarrhea, constipation, palpable abdominal mass, increasing abdominal girth, digestive disorders, melena or pseudo-ascites. Only a minority of ICLs are asymptomatic and detected incidentally [1-3, 6]. Due to the multitude of symptoms diagnosis can be challenging [7]. The great variety of differential diagnoses of ICL include many benign and malignant tumors [3].

Despite the fact that septated intraabdominal fluid may be an indicator for ICL, the definite diagnosis is established after surgical resection and subsequent histopathological examination of the tissue $[3,8]$. As malignancy has to be excluded and ultrasonography (US), computed tomography (CT) or magnetic resonance tomography (MRT) are very sensitive, yet insufficient specific diagnostic tools for making the diagnosis, surgery is the only method to confirm ICL [3]. We describe a single center experience of ICL during the years 2009 - 2011 pointing out the difficulties of making the diagnosis and the value of US.

\section{Case Reports}

\section{Case 1}

A 13-month-old girl was first seen by a practicing pediatrician because of a disposition for constipation persisting 

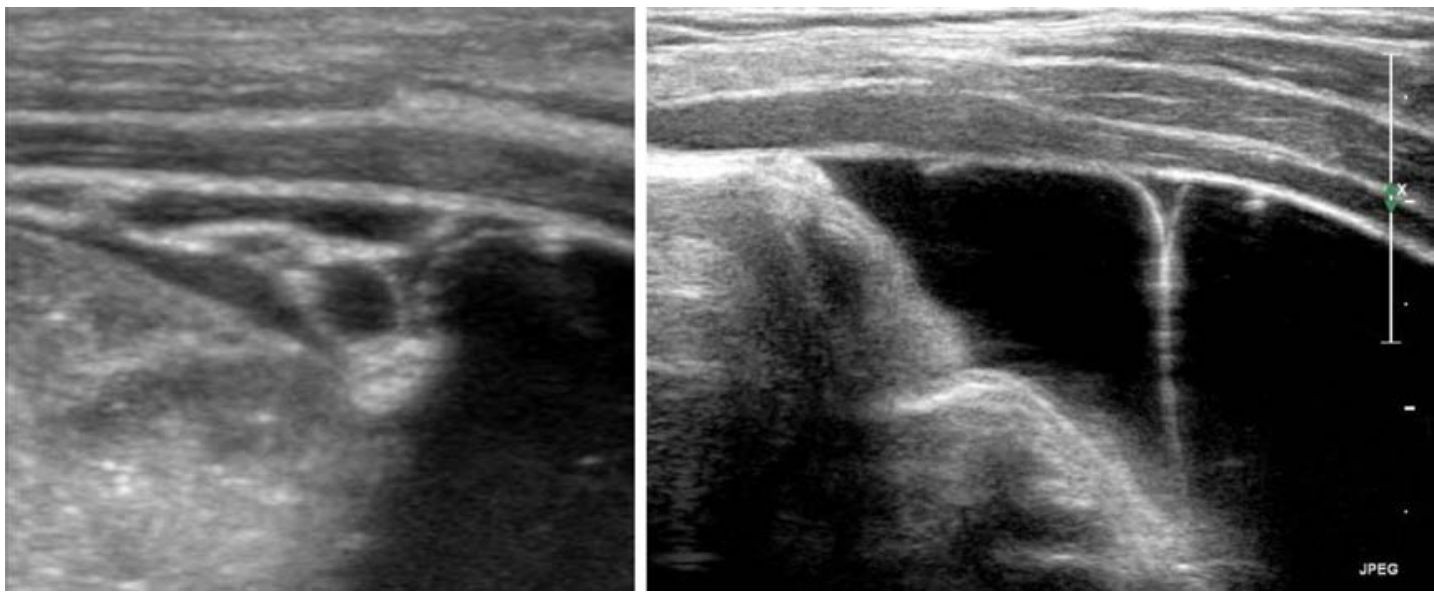

Figure 1. Intraabdominal cystic mass with internal septations in patient 1.

over a period of several months and a failure to thrive with a corresponding body weight below the third percentile. A comprehensive clinical investigation and laboratory test showed no abnormalities. The initial US showed ascites of unknown etiology, and further follow-up sonographies at the hospital confirmed this, showing now for the first time echogenic contents within the intraabdominal fluid accumulation. Complementary laboratory tests (immunoglobulins, antibodies against human tissue transglutaminase (tTG), iron metabolism, liver and pancreas enzymes, folic acid, vitamin B12, vitamin D, serum protein, serum electrophoresis, coagulation status, infection parameters, bacteriological stool test, calprotectin, and alpha-1-antitrypsine), as well as gastroscopy did not result in additional diagnostic findings. Two months later, a well defined cystic mass with internal septations was demonstrated via US (Fig. 1) in the area of the intraabdominal fluid accumulation, leading to the differential diagnosis of a mesenterial cyst or an ICL for the first time. As further imaging techniques were not considered to give additional information in this case, explorative laparotomy followed and a lymphatic cyst with a diameter of

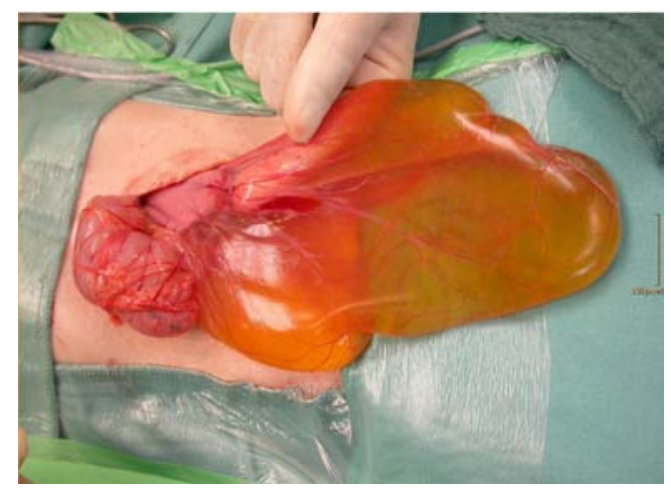

Figure 2. ICL (diameter $13 \mathrm{~cm}$ ) originating from the ligamentum gastrocolicum in patient 1 .
$13 \mathrm{~cm}$, originating from the ligamentum gastrocolicum, was extirpated completely (Fig. 2). The histological examination confirmed the existence of an ICL. After surgery the patient gained weight appropriate to age and showed no relapse up to 2013 .

\section{Case 2}

The 28-month-old boy was admitted to a tertiary care hospital because of recurrent vomiting since 1 day. He neither had diarrhea nor abdominal pain. To exclude invagination an US was performed showing free abdominal fluid and a slight thickening of the small intestine wall but no specific signs of invagination. Finally, a stool investigation identified norovirus as the causative agent of vomiting. Laboratory test did not reveal any abnormalities and sonographic follow-up examinations demonstrated persistent free intraabdominal fluid with no tendency to regression. As a consequence, further differential diagnostic workup was initiated comprising complementary laboratory tests (urine test, serum immunglobulins, aldosterone, stool-osmolality and stool electro-

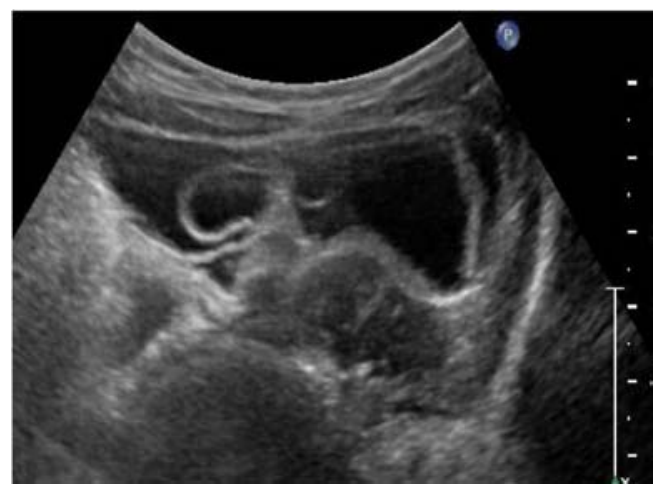

Figure 3. Intraabdominal cystic mass with internal septations in patient 2. 


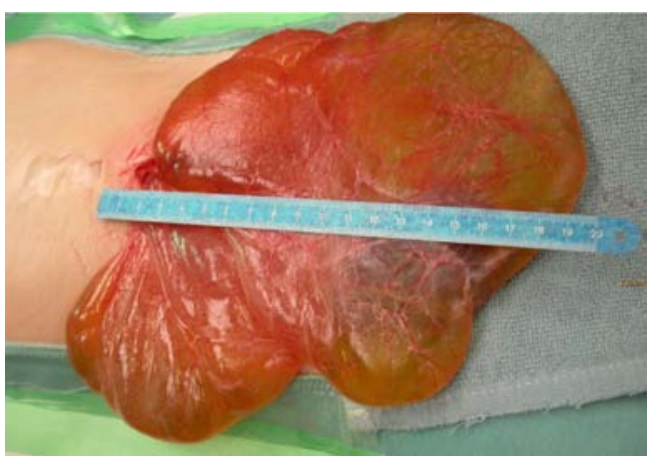

Figure 4. ICL (diameter $20 \mathrm{~cm}$ ) originating from the ligamentum gastrolienale in patient 2.

lytes), echocardiography and portal vein sonography, but it did not result in additional diagnostic findings. During the observation period the patient always showed an excellent health status and no failure to thrive with a constant body weight along the 90th percentile. After 3 months of monitoring, US demonstrated septations in the ascites for the first time (Fig. 3), now extending over four quadrants from the small pelvis up to the lienorenal space. To exclude malignancy the remaining diagnostic possibility was a needle aspiration of the fluid. However, the subsequent puncture of the ascites did not provide any additional information. The result of the cytologic examination was an exudate, free of atypical cells.

Furthermore, an MRT was made, confirming the result of the US: four-quadrant ascites with septations but also a partial displacement of intestinal structures, which could not be detected by US so far. However, a defined cystic structure in the area of the ascites could neither be demonstrated by MRT nor by US. After having completed the differential diagnostic workup, the most likely diagnosis remained an ICL so that surgical intervention to remove the space-occupying lesion by explorative laparotomy was decided. Histologically an ICL with a diameter of $20 \mathrm{~cm}$, originating from the ligamentum gastrolienale, was confirmed (Fig. 4).

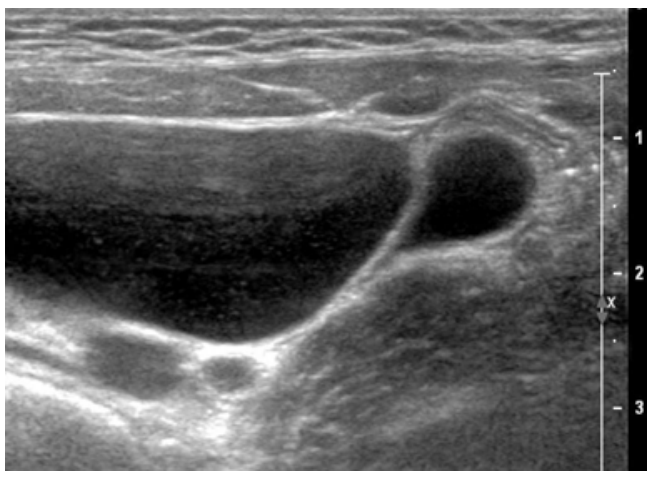

Figure 5. Thin-walled, fluid-filled cystic lesions in the left hemi-abdomen in patient 3.

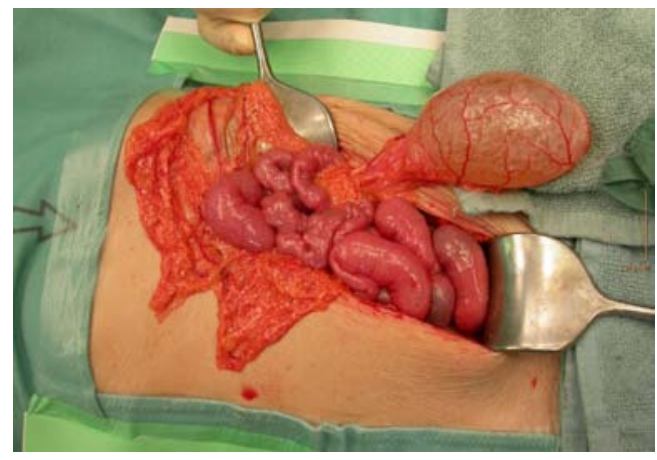

Figure 6. ICL (diameter $7 \mathrm{~cm}$ ) originating from the omentum majus in patient 3 .

\section{Case 3}

In a 5 years and 1 month old girl a left-sided ureteropelvic junction stenosis with a consecutive hydronephrosis type IIIIV was diagnosed in a tertiary care hospital. For urological examination and renal scintigraphy the patient was transmitted to the university hospital. US was repeated, showing numerous, thin-walled, partly confluent fluid-filled lesions in the left hemi-abdomen extending into the small pelvis (Fig. 5). Based on the experience of former cases the differential diagnosis of an ICL was taken into account early. Furthermore, another US of the abdomen, CT and MRT were added, especially to evaluate the renal situation and a possible correlation between the cystic formations and the ureteropelvic junction stenosis. After this, the indication for laparotomy was raised. During surgery, an ICL with a diameter of 7 $\mathrm{cm}$, originating from the omentum majus, was completely removed (Fig. 6). A correlation between the cystic spaceoccupying lesion and the left-sided ureteropelvic junction stenosis was excluded. Due to the both-sided normal renal function, there was no indication to operate the ureteropelvic junction stenosis at the same time so that a pyeloplasty was planned later on. In the follow-up examinations after surgery, the patient presented in age-corresponding good condition.

\section{Discussion}

ICLs originating from the small or large intestine are rare and due to slow development the overwhelming majority of patients remain asymptomatic over a certain period, leading to diagnosis mainly during childhood and typically beyond the neonatal phase $[5,6,9]$. Seventy percent of ICLs originate from the mesentery, $15 \%$ from the omentum (omentum majus, minus, and ligamentum gastrocolicum), 10\% from the mesocolon and only $5 \%$ from the retroperitoneal space $[4,6]$. Depending on the origin of the ICL, different clinical presentations can appear.

Fifty-nine percent of patients with ICL show unspecific 
intestinal disorders, only $17 \%$ with acute abdominal symptoms whereas the remaining part of ICL is detected incidentally [10].

The unspecific abdominal symptoms are usually observed, ranging from an increasing abdominal girth, palpable mass, diarrhea, constipation, nausea, vomiting, digestive disorders and melena to pseudo-ascites [4, 9]. Acute abdominal symptoms mainly result from complications caused by ICL comprising fever, nausea, vomiting and peritonism in case of infection, or ileus in case of obstruction. A rupture, hemorrhage or torsion of a cyst may also lead to acute symptoms $[2,10]$. Especially mesenteric lymphangiomas seem to become apparent more frequently by acute abdominal symptoms, particularly through an obstruction of the surrounding intestine or symptoms of pseudo-appendicitis whereas omental lymphangiomas are responsible for the voluminous pseudo-ascites without clinical symptoms. Retroperitoneal lymphangiomas remain asymptomatic for the longest whereas mesocolic lymphangiomas mostly present as a fixed tumor mass causing pain and appendicitis-like symptoms in the right lower abdomen [4]. In our case series two patients showed unspecific abdominal symptoms like constipation and vomiting, and in one child the ICL was detected incidentally during a routine US. The abdominal symptoms in our first patient with constipation and a body weight below the third percentile might have been caused by the underlying ICL, since displacement or obstruction of surrounding intestinal loops or organs through ICL has been reported previously [3]. The cystic lesion could have had a compressing effect on the bowel with the consequence of constipation and loss of appetite. In this case, repeated US was able to demonstrate a cystic structure in the primarily assumed ascites. The origin of our first patient's ICL was the ligamentum gastrocolicum (part of omentum), which initially also presented with pseudo-ascites, as described in the study of Galifer et al [4].

Hypothetically, the patient was asymptomatic over a longer period because of the slow progression of the ICL. After reaching a diameter of $13 \mathrm{~cm}$ the cystic lesion might have become symptomatic by compression or lateral displacement of intestinal loops. In our second patient, the ICL originated from the ligamentum gastrolienale (part of omentum), also mimicking an extensive pseudo-ascites initially. As the patient was in best health status and free of any abdominal symptoms after recovering from the norovirus infection despite the persistent intraabdominal fluid accumulation, vomiting might have been caused by infection and not by the ICL. In our asymptomatic third patient the diagnosis of an ICL was made incidentally within a routine US reevaluating a preexisting hydronephrosis. A correlation between the ICL and hydronephrosis was excluded during surgery. In patients with the suspicion of an ICL, traumatic cysts, pseudo-cysts, intestinal duplication cysts, dermoid cysts and cysts of the urogenital tract have to be taken into account as differential diagnoses [3, 4, 7]. Our case series underlines, despite multiple radiological investigations, the difficulty of getting rapid and adequate diagnosis, especially in case of pseudo-ascites without clinical symptoms. The main role of imaging in ICL is to present the cystic nature of the assumed pseudo-ascites or abdominal masses [4, 6, 8, 11]. Sonography, with a sensitivity of $85 \%$ is superior to CT in demonstration of septations and the internal nature of the cysts. Especially sonographic detection of septations in the ascites (demonstrable in $85 \%$ of ICL), of defined chambered cysts (in $15 \%$ of the cases) or echogenic contents in the cysts (in 50\% of the cases) are highly suggestive signs that might lead to the important differential diagnosis of an ICL $[8,9$, 11]. In all of our three patients the sonographic demonstration of septations in the ascites succeeded, whereas cystic structures could only be presented in patients 1 and 3. In our second patient the cystic nature was finally detected by surgery. So, US is able to demonstrate the cystic nature, location and limits of the abdominal mass; however, interpretation of these results to the correct presurgical diagnosis remains difficult $[3,4,8]$. In accordance with Lorken et al, MRT might be indicated to get detailed information about the origin and extent of a lesion or an infiltration of surrounding structures so that some authors consider this imaging method as gold standard in diagnosing ICL [3]. However, as seen in this case series, MRT did not always provide additional findings to sonography as reported in previous cases in literature $[3,6$, 8]. A puncture of the space-occupying lesion or the assumed ascites for diagnostic purposes is not recommended, considering the spread of a potentially existing malignant tumor [6]. Especially if the cystic nature of the space-occupying lesion cannot be detected immediately and if an unclear ascites persists, as reported in our second patient, puncture and cytologic examination might be considered in individual cases after precise evaluation $[6,12]$. The classic histological presentation of ICL shows a flat, endothelial lining of the cyst wall, bands of smooth muscle fibers in the wall layers as well as lymphatic cells in the vascular cavities $[3,10]$. Misleadingly, mesenterial cysts and ICL are often classified together. The differentiation between these two types of cysts, however, is of prognostic relevance due to a recurrence risk in case of incomplete resection of an ICL [1, 3, 13, 14]. Due to an adverse anatomic localization, incompletely removed ICL often results in a progressive, infiltrative growth in the relapse phase, and this might also require the additional removal of the involved intestinal or organ segments [9]. Therefore, the only curative therapy is a complete surgical resection of the space-occupying lesion [2]. The risk of recurrence after complete resection has been reported between $0 \%$ and $14 \%$ and malignant degeneration is rare [3]. Up to 2013 all our patients present in good clinical condition without relapse. In conclusion, because of the rarity and often incidental detection of this disorder, the widespread clinical picture and the lack of helpful laboratory analyses, high 
suspicion and awareness with regard to this disease is necessary to shorten diagnostic workup and avoid unessential invasive examinations. According to our experience and the review of the literature, one might use US as the only imaging technique especially when septations, cysts or echogenic contents within the ascites are observed. In view of this fact the routine use of US in children with unspecific abdominal symptoms seems to be justified [7].

\section{Abbreviations}

ICL: intraabdominal cystic lymphangioma; US: ultrasonography; CT: computed tomography; MRT: magnetic resonance tomography; tTG: human tissue transglutaminase

\section{References}

1. Eckert K, Vossough A, Michna, D, Zirbes, T, Liedgens, P. Stielgedrehtes, intraabdominales Lymphangiom. Monatsschrift Kinderheilkd. 2008;157:368-371.

2. Morger R, Bolliger B, Muller M. [Abdominal lymphangioma in childhood]. Padiatr Padol. 1991;26(1):31-34.

3. Lorken M, Marnitz U, Manegold E, Schumpelick V. [Intra-abdominal lymphangioma]. Chirurg. 2001;72(1):7277.

4. Galifer RB, Pous JG, Juskiewenski S, Pasquie M, Gaubert J. Intro-abdominal cystic lymphangiomas in childhood. Prog Pediatr Surg. 1978;11:173-238.

5. Goh BK, Tan YM, Ong HS, Chui CH, Ooi LL, Chow $\mathrm{PK}$, Tan CE, et al. Intra-abdominal and retroperitoneal lymphangiomas in pediatric and adult patients. World $\mathrm{J}$
Surg. 2005;29(7):837-840.

6. Witzel K, Kronsbein H, Pleser M, Hunfeld H, Rumpf KD. [Intraabdominal cystic lymphangioma in childhood. Report of 2 cases]. Zentralbl Chir. 1999;124(2):159-162.

7. Shafi SM, Malla MA, Reshi FA. Giant primary omental cyst mimicking a pseudoascites. Afr J Paediatr Surg. 2009;6(1):58-60.

8. Vargas-Serrano B, Alegre-Bernal N, Cortina-Moreno B, Rodriguez-Romero R, Sanchez-Ortega F. Abdominal cystic lymphangiomas: US and CT findings. Eur J Radiol. 1995;19(3):183-187.

9. Chang TS, Ricketts R, Abramowsky CR, Cotter BD, Steelman CK, Husain A, Shehata BM. Mesenteric cystic masses: a series of 21 pediatric cases and review of the literature. Fetal Pediatr Pathol. 2011;30(1):40-44.

10. Steyaert H, Guitard J, Moscovici J, Juricic M, Vaysse P, Juskiewenski S. Abdominal cystic lymphangioma in children: benign lesions that can have a proliferative course. J Pediatr Surg. 1996;31(5):677-680.

11. Moritz J, Riedel C, Bolte H. Abdominelle zystische Raumforderungen in der Kinderradiologie. RoFo Fortschritte auf dem Gebiet der Rontgenstrahlen und der Bildgeb. Verfahren. 2006;178:13.

12. Perriello VA, Jr., Flemma RJ. Lymphangiomatous omental cyst in infancy masquerading as ascites. J Pediatr Surg. 1969;4(2):227-230.

13. Stopinski J, Stephan S, Staib I. [Intra-abdominal cystic lymphangioma and mesenteric cysts as a cause of abdominal discomfort]. Langenbecks Arch Chir. 1994;379(3):182-187.

14. Kosir MA, Sonnino RE, Gauderer MW. Pediatric abdominal lymphangiomas: a plea for early recognition. J Pediatr Surg. 1991;26(11):1309-1313. 